\title{
Étienne-Gabriel MORELLY, Code de la nature, édition critique par Stéphanie ROZA
}

Paris, La ville brûle, 2011, 174 p.

\section{Claude Mazauric}

\section{(2) OpenEdition \\ 12 Journals}

\section{Édition électronique}

URL : https://journals.openedition.org/ahrf/12656

DOI : $10.4000 / a h r f .12656$

ISSN : 1952-403X

Éditeur :

Armand Colin, Société des études robespierristes

\section{Édition imprimée}

Date de publication : 1 septembre 2012

Pagination : 176-178

ISBN : 978-2-200-92761-5

ISSN : 0003-4436

Référence électronique

Claude Mazauric, «Étienne-Gabriel morelly, Code de la nature, édition critique par Stéphanie roza », Annales historiques de la Révolution française [En ligne], 369 | juillet-septembre 2012, mis en ligne le 28 février 2013, consulté le 22 avril 2022. URL : http://journals.openedition.org/ahrf/12656 ; DOI : https:// doi.org/10.4000/ahrf.12656

Ce document a été généré automatiquement le 22 avril 2022.

Tous droits réservés 


\title{
Étienne-Gabriel MORELLy, Code de la nature, édition critique par Stéphanie ROZA
}

Paris, La ville brûle, 2011, 174 p.

\author{
Claude Mazauric
}

\section{RÉFÉRENCE}

Étienne-Gabriel MORELLY, Code de la nature, édition critique par Stéphanie ROZA, Paris, La ville brûle, 2011, 174 p., 978-2360120147, $14 €$

Paru en 1755, le Code de la nature est un ouvrage tout à fait essentiel pour qui s'interroge sur ce que l'on appelait naguère le « pragmatisme » des Lumières : c'est-à-dire non pas une élémentaire mise en projet de simples programmes antérieurement pensés, comme le croient ceux qui rapetissent tout ce qu'ils touchent, mais la recherche d'une forme d'énonciation de ce que l'on pourrait entreprendre qui modifierait radicalement l'ordre des choses et $\mathrm{du}$ monde. Les constructions discursives, qui rendent compte de l'imaginaire transformateur de l'ordre social où se dévoile ce que l'on désigne d'ordinaire comme des " utopies ", ressortissent de ce champ à la fois philosophique et littéraire où se révèle tout autant une poétique prophétique qu'une série d'énoncés de type juridique, institutionnel ou politique. Le Code de Morelly répond entièrement à ce dernier schéma et peut même être considéré comme un modèle de ce genre de textes qui firent le bonheur de nombre de penseurs et de lecteurs dans la seconde moitié du XVIII ${ }^{e}$ siècle : on ne s'étonnera donc pas de ses rééditions successives et de la célébrité consécutive du nom de son auteur dont on savait pourtant peu de choses. Mais comme l'on ne prête qu'aux riches, «on» (le public) n'hésita pas à tenir que Morelly n'était qu'un pseudonyme, l'auteur de ce grand texte ne pouvant être qu'un grand "philosophe ", La Beaumelle peut-être, plus certainement Denis Diderot dont chacun savait qu'il avait encouragé un autre grand subversif, Jean-Jacques Rousseau, le 
« citoyen de Genève ", à rédiger son premier discours couronné en 1750 par l'Académie de Dijon. C'est que le Code de la nature sentait le soufre! Dans un fort développement de sa présentation consacrée à la réception de l'ouvrage, Stéphanie Roza le rappelle opportunément. Babeuf lui-même, au Procès de Vendôme en 1797, attribuera encore à Diderot la paternité de l'œuvre majeure de Morelly.

2 La réédition de ce grand ouvrage que nous propose Stéphanie Roza, brillante et jeune philosophe autant qu'historienne attentive à bien identifier les sources de son enquête, se signale par la précision de ses analyses et la haute idée qu'elle se fait d'un texte tout à fait représentatif des audaces philosophiques du siècle dit des «Lumières ». Dans son «Introduction» (42 pages denses), Stéphanie Roza rappelle à juste titre que le chercheur écossais R. C. Coe avait établi en 1957 non seulement que Étienne Gabriel Morelly était l'auteur de l'ouvrage en question, mais qu'il n'était pas un médiocre folliculaire sorti de rien, se signalant au contraire comme l'auteur de textes qui connurent une notoriété non négligeable dès leur temps. Stéphanie Roza, sur ce plan, complète fort bien ce que nous savions de lui, même si beaucoup de données sociobiographiques touchant à Morelly demeurent encore très floues. Stéphanie Roza montre en particulier tout l'intérêt de l'autre texte de Morelly qui a contribué à fonder sa notoriété posthume, à savoir son roman publié anonymement deux ans avant le Code, en 1753, Naufrage des isles flottantes ou Basiliade du célèbre Pilpai (Pilpai étant le nom d'un supposé philosophe indien) : ici l'utopie n'a plus rien d'insulaire comme c'était le cas dans la célèbre Utopia de Thomas More, parue en 1516 et rééditée plusieurs fois au $\mathrm{XVIII}^{\mathrm{e}}$ siècle, puisque c'est au contraire l'occident européen qui est devenu un semis d'îles égarées tandis que le lieu de la nouvelle Salente, heureuse et régénérée, est présenté comme un continent... La signification profonde du Code, paru peu après la Basiliade, s'éclaire de leur confrontation: le plan de législation imaginé par Morelly n'était pas un dispositif adapté à une situation marginale et exceptionnelle, mais au contraire se voulait un projet majeur de réorganisation de l'ordre social dominant, fondé sur la proposition que la «communauté des biens doit devenir la matrice de l'organisation sociale» face à «la propriété privée » qui en serait son « détournement ».

Le Code de la nature relève donc moins de la poétique que de la "théorie sociale" comme l'affirme sans hésitation Stéphanie Roza. L'exégèse à laquelle se livre l'éditrice du texte lui-même, la conduit à mettre en valeur le champ conceptuel dans lequel il s'inscrit: la vision historiciste de l'état présent de la condition humaine, les fondements philosophiques modernes du projet de réorganisation de la société, les domaines d'intervention de la "législation idéale»; le rejet de la perspective « contractualiste» comme résultante d'une décision raisonnée des hommes socialisés se double de la reconnaissance d'une nécessité de «sociabilité » quasiment naturelle, inscrite dans la recherche de leur bien-être par les hommes eux-mêmes, etc. Stéphanie Roza en montre aussi les limites bien connues : la faiblesse de la réflexion économique, la quasi-ignorance des conflits sociaux, l'optimisme illusionniste de l'auteur : Morelly n'était pas Mably! Il n'était pas non plus Rousseau dont l'anthropologie éminemment subversive, si fortement exposée dans le second discours paru comme le Code en 1755, se définit à l'opposé de celle de Morelly qui est continuiste et processive. Mais Stéphanie Roza défend cependant l'hypothèse d'une "proximité morale ", j'ajouterais idéologique, entre les deux "plébéiens» contemporains que furent le citoyen de 
Genève et le roturier champenois, sujet du roi de France, quoiqu'ils ne se fussent jamais rencontrés.

Reste à découvrir, amis lecteurs, si vous ne l'avez déjà fait, les quatre parties du Code de la nature : l'annotation à la fois fine, légère et précise de l'édition proposée en facilitera la lecture et la compréhension distanciée. On rappellera évidemment que la construction imaginée dans la seconde partie du traité a inspiré nombre de penseurs de l'alternative utopiste postérieure comme Cabet, Fourier, Dézamy, Morris... et bien d'autres depuis, jusque dans les révolutions contemporaines. Comment faut-il apprécier cette démarche? Est-on confronté à l'énoncé d'une utopie communiste? Socialiste? Stéphanie Roza retient les deux acceptions. L'introduction porte le titre suivant : « Un programme socialiste au siècle des Lumières " mais la conclusion voit en Morelly «un précurseur du communisme». Aujourd'hui, dans la configuration politique et idéologique de notre temps, après la riche et terrible histoire du $\mathrm{XX}^{\mathrm{e}}$ siècle, la fameuse bataille d'idées qui fit rage hier, opposant la perspective du communisme à la révolution socialiste, celle-ci étant soit la première étape de la marche au communisme qui incarnerait la visée fondatrice, ou bien le masque réformiste et régressif d'une renonciation à l'idée de transformation radicale de l'ordre social, cette controverse a perdu le plus fort de son acuité. Reste cependant à prendre la mesure d'un fait essentiel : toute critique radicale, pratique et théorique, des sociétés de classes fondées sur le primat de l'appropriation privée des survaleurs produites par le travail social, implique l'idée alternative de la communauté des biens et des travaux, ce qui établit celle-ci comme la matrice en perspective du communisme. Le reste relève de l'histoire, de la philosophie et de la politique. 